\title{
Melanin from the lichen Lobaria pulmonaria: physicochemical properties and biological activity
}

Rassabina A.E. ${ }^{1 *}$, Khabibrakhmanova V.R. ${ }^{1,2}$, Leksin I.Y. ${ }^{1}$, Minibayeva F.V. ${ }^{1}$

${ }^{1}$ Kazan Institute of Biochemistry and Biophysics, FRC Kazan Scientific Center of the RAS, Kazan, Russia

${ }^{2}$ KNITU, Kazan, Russia

*email: AERassabina@yandex.ru

Symbiotic lichens are of a great interest for research, as they display high stress resistance. Currently, the chemical composition of many lichens has been determined, and some metabolites involved in the response to stresses have been identified. Melanins can protect lichens from UV radiation and high light, however their chemical structure, physicochemical properties, and genes responsible for melanin synthesis in lichens are not studied yet. So far, only a limited number of lichens, including Lobaria pulmonaria, have their genome sequenced. We studied the structure, elemental composition, functional groups, paramagnetic and antioxidative properties of UV inducible melanin in L. pulmonaria and correlated these parameters with expression of the genes involved in melanin biosynthesis. According to the chemical structure of the monomer link, these high-molecular weight pigments are classified into eumelanin, allomelanin, pheomelanin and other types. Melanin extracted from L. pulmonaria contains C 49.6\%, $\mathrm{O} 40.2 \%, \mathrm{H} 5.8 \%, \mathrm{~N} \mathrm{4.4 \%}$. Using UV and FTIR spectroscopy, we showed similarity of its chemical properties to those of the commercial eumelanin from Sepia officinalis. When studying the paramagnetic properties of extracted melanin by EPR spectroscopy, a signal corresponding to the free electron signal was registered, and the content of paramagnetic centers was determined as $0.64 \times 10^{17} \mathrm{spin} / \mathrm{g}$. Lichen melanin showed antioxidative activity against the DPPH radical with $\mathrm{IC}_{50} 273 \mu \mathrm{g} / \mathrm{ml}$. Interestingly, upregulation of genes responsible for both eumelanin and DHN melanin was found in L. pulmonaria in response to UV-B exposure. These are genes encoding tyrosinases, multicopper oxidases for eumelanin and polyketide synthases, THN-dehydrogenases, scytalon dehydrogenases for DHN melanin. In conclusion, melanins synthesized by extremophilic lichens protect against UV radiation, oxidative stress, intoxication by heavy metals and xenobiotics. Therefore, lichen melanins have clear biotechnological perspectives in bioremediation and pharmacy.

Acknowledgements: The work was supported by the RSF grant (No. 18-14-00198) and the RFBR grant "Postgraduates" (No. 20-34-90044). 